\title{
The Design and Regulation of High Frequency Traders
}

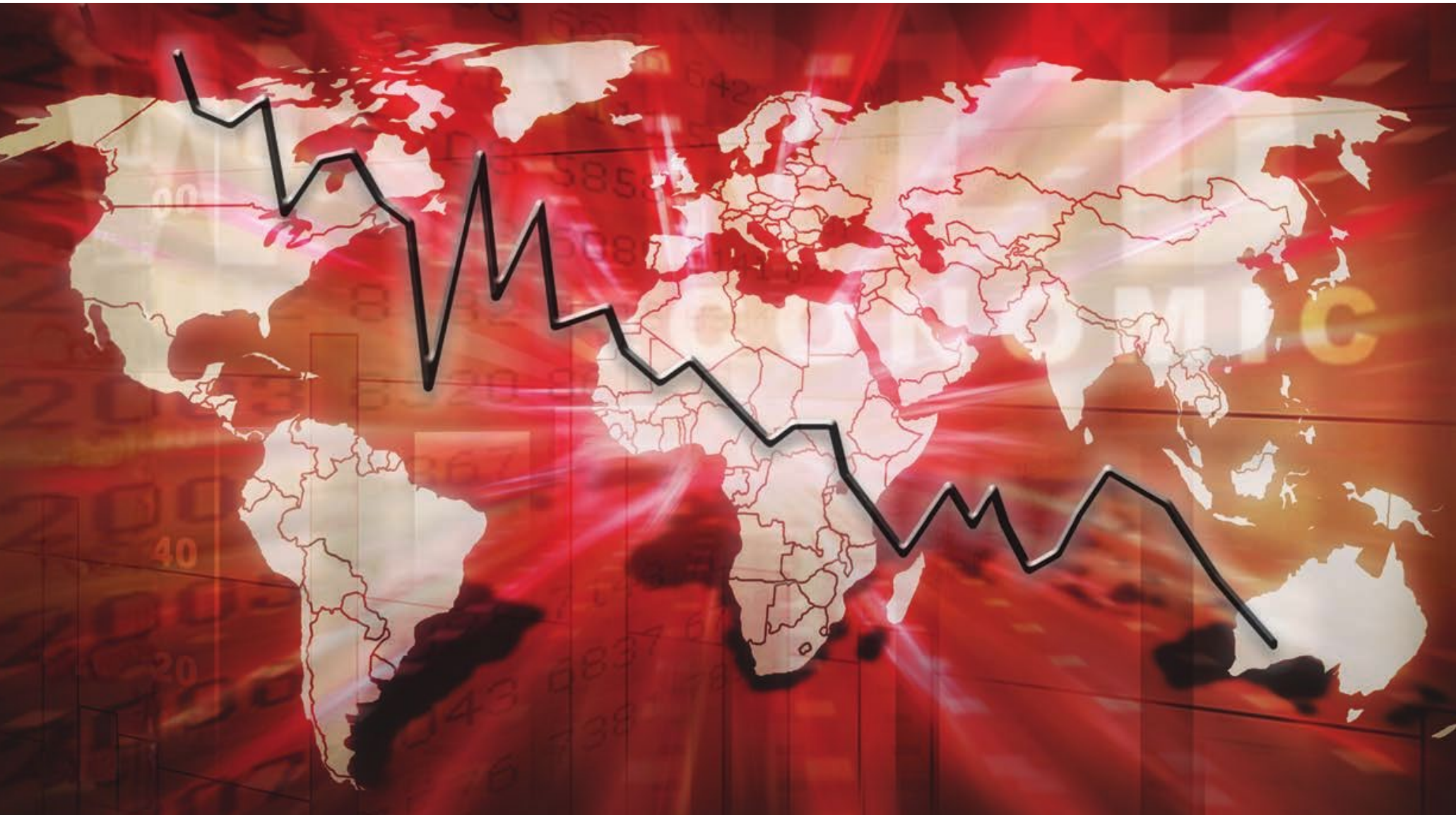

Professor Daniel Ladley, University of Leicester

Working Paper No. 1902 March 2019 


\title{
The Design and Regulation of High Frequency Traders
}

\author{
Daniel Ladley* \\ University of Leicester School of Business \\ University of Leicester \\ LE17RH \\ UK
}

March 29, 2019

\begin{abstract}
Central to the ability of a high frequency trader to make money is speed. In order to be first to trading opportunities firms invest in the fastest hardware and the shortest connections between their machines and the markets. This, however, is not enough, algorithms must be short, no more than a few lines of code. As a result there is a trade-off in the design of optimal HFT strategies: being the fastest necessitates being less sophisticated. To understand the effect of this tension a computational model is presented that captures latency, both of code execution and information transmission. Trading algorithms are modelled through genetic programmes with longer programmes allowing more sophisticated decisions at the cost of slower execution times. It is shown that depending on the market composition short fast strategies and slower more sophisticated strategies may both be viable and exploit different trading opportunities. The relative profits of these different approaches vary, however, slow traders benefit from their presence. A suite of regulations are tested to manage the risks associated with high frequency trading, the majority are found to be ineffective, however, constraining the ratio of orders to trades may be promising.
\end{abstract}

Keywords: Finance, Genetic Programming, High Frequency Trading, Strategy Design, Regulation

*E-mail: d.ladley@le.ac.uk, Telephone: +44 (0)116 252 5285. I thank the British Academy and Leverhulme Trust for their support through the Small Grant scheme. In addition I thank seminar participants at Computing in Economics and Finance, 2017, International Finance and Banking Association, 2017, King's College London and the University of Leicester. 


\section{Introduction}

The phrase 'time is money' neatly captures the business model of high frequency trading (HFT). These traders make money by being the first, whether that's the first to trade against incoming orders or the first to revise stale quotes in the event of changing market conditions. Slower algorithms miss out on the best opportunities and bare more risk. As a result there is an arms race amongst HFT firms to create algorithms that can identify mispricings and execute new orders in the fastest time. The time to execute an order depends on several factors: the time taken for signals to travel between the exchange and the HFT computers, the computer hardware and the algorithms that run on them. HFT firms pay to minimise the first two factors and not be at a disadvantage to their competitors. Co-locating of their hardware with that of the exchange ${ }^{1}$ and purchasing the fastest and most up to date computer hardware. The final component, the algorithms that govern trade, is the source of competitive advantage for HFT firms but also represents a trade-off. Longer trading algorithms take more computational cycles to execute and therefore result in slower actions. By reducing the length of an algorithm the HFT firm makes their order more likely to be first. Shortening algorithms, however, has a consequence reducing the number of lines of code reduces the information processing capacity of the algorithm - reducing the algorithms ability to identify profitable opportunities and avoid losses. This is a fundamental trade off in the design of HFT algorithms. The shorter the algorithm the more likely it is to be first to act but the less sophisticated its strategy.

It is this trade-off that will be the basis of the investigation in this paper. A model is constructed of the behaviour of HFT algorithms subject to a speed/sophistication trade-off. HFT traders endogenously decide when to trade based on the actions of others and information arriving at the market. I analyse the problem faced by HFT strategy designers - what is the optimal strategy when speed may be traded-off against information processing ability. This issues has not previously been considered in the literature. Whilst multiple papers have looked at trading speed in the face of technological costs (see for instance Biais et al., 2015 or Delaney, 2018), they have done so in an environment of perfect rationality. Here I make a unique argument that the cost of speed is not just monetary but also in terms of cognitive (computational) sophistication, i.e. in order to increase speed perfect rationality has to be sacrificed. The extent to which traders are willing to do this is not clear. For instance it is not inevitable that algorithms will be ever simpler and faster. Unsophisticated trading algorithms may leave money on the table that slower and more sophisticated HFT's may identify and capture.

In order to investigate this question it is necessary to have a representation of strategies where computational sophistication is related to time in a realistic manner. The natural choice for this is to use a computational approach in which high frequency traders are, like real life, algorithms. Longer algorithms, as measured by the number of lines of code, generally take more time to execute as the computer processor must step through each in turn. ${ }^{2}$ The difficulty of this approach is then specifying the optimal trading algorithm(s). The relationship between code length, algorithm design and performance is complex and non-tractable. To resolve this

\footnotetext{
${ }^{1}$ To facilitate this many exchanges offer space on their own site with guaranteed lengths of wire between machines to create a level playing field amongst.

${ }^{2}$ There are exceptions here, conditional statement such as if $X$ do $Y$ can disproportionately slow down execution as they limit the processors ability to cache future actions.
} 
I numerically optimise the trading algorithms by competing them against each other within a market. Maintaining those that do well whilst continuously looking for modifications and improvements that will enhance performance. This approach of optimising programmes (as opposed to parameters) is referred to as genetic programming - essentially evolving algorithms to solve a task. ${ }^{3}$ For our problem this creates an attractive analogy: a market of trading algorithms competing to make profits based on their speed and sophistication with the most successful surviving and the losses being replaced. Given sufficient time this process will lead to the identification of a steady state in which important details of trading algorithms and market behaviour no longer change. It is this state, rather than the optimisation process which produces it that I will analyse. Genetic programming has been used to simulate trading strategies of different sophistication's before. Yeh (2008) uses a genetic programming model to show that greater intelligence improves market efficiency. Ladley et al. (2015) use a genetic programming model to investigate the relationship between skill and market fragmentation and show that large numbers of unskilled individuals make the market more susceptible to shocks. Whilst Manahov et al. (2014) shows that varying the length of trading strategies impacts trader and market performance. Importantly, however, no work has looked at the trade-off between speed and sophistication.

Using the model I show that despite competition to be fastest and therefore first to arrive at a trading opportunity not all HFT's take this approach in equilibrium. Whilst increasing competition between HFT's does push the population towards shorter strategies this is not universal. Some traders adopt longer strategies and are shown to generate greater per order (and per trade) profits than those adopting the fastest algorithms. These traders identify and exploit trading opportunities that their faster competitors miss. As such there are multiple equilibria in the design of HFT trading strategies.

The presence of HFT's within the market is shown to be generally positive. Increasing numbers of HFT's increase market quality through higher liquidity and lower pricing errors. At the same time they have relatively little effect on the overall profits of slow traders, instead their profits come through improved prices and reduced waiting costs for trade. The greater competition coming from higher numbers of HFT's leads to reducing profits for this group but no negative effects for slower traders.

The effectiveness of a suite of regulations proposed to manage the impact of high frequency traders is considered. The majority, including minimum resting times, speed bumps and increased tick sizes are found to have little or no positive effect, often damaging market quality and increasing the returns of HFT's at the expense of slower traders. Transaction taxes are found to be particularly detrimental to both the market and traders. The only regulation which potentially has a positive effect are constraints on the ratio of trades to orders submitted by the HFT's. This regulation increases the profits of slow traders relative to HFT's but at the expense of reducing market liquidity.

The remainder of the paper is organised as follows. Section 2 considers previous work looking independently at trading speed and the effect of cognitive ability on market performance together

\footnotetext{
${ }^{3}$ Genetic Programming has been used in papers for various finance problems. Neely et al. (1997) use the approach to find technical trading rules, Chen and Wang (2015) use it to construct portfolios, whilst Lensberg et al. (2015) and Yeh and Yang (2010) amongst others use this approach to simulate markets.
} 
with HFT regulation. Section 3 present a model of a market in which traders trade-off speed and strategic sophistication. Section 4 presents results showing optimal trading strategies and market quality whilst Section 5 looks at the effectiveness of regulations. Section 6 concludes.

\section{Related Literature}

The trade-off between speed and computational time has not previously been considered in relation to trading. There have, however, been pieces of work that have looked at each factor individually. The link between intelligence and financial success has been studied extensively. Some of the earliest work looked at the connection between wages and intelligence (see for instance Moore, 1911). More recently papers have considered the link between cognitive ability and stock market performance. Grinblatt et al. (2012) for instance shows that higher IQ leads to better trading performance whilst Coval et al. (2004) shows that even in the absence of superior information some traders are able to consistently outperform others, in other words they are skilled.

The reasons for this out performance may be many fold and have been studied experimentally. Burks et al. (2009) showed that more intelligent players can better predict their opponents actions in two player sequential games whilst Corgnet et al. (2015) find that high intelligence traders are more likely to identify points at which the asset is under priced or overpriced compared to their low cognitive ability compatriots. Chen et al. (2017) analyses which aspects of intelligence are responsible for the higher returns of traders and find that this varies depending on the types of individuals they are competing against. The effect on the market of cognitive ability has also received some attention. Breaban and Noussair (2015) show experimentally that higher cognitive ability results in prices being closer to fundamental values, while Noussair et al. (2016) show that in an experimental setting more intelligent individuals lead to a closer relationship between the fundamental and market price.

High frequency trading has received a great deal of attention in recent years from both theoretical and empirical analysis. Papers have considered liquidity (Hendershott et al., 2011), price discovery (Brogaard et al., 2014) and strategy effects (Hagströmer and Nordén, 2013) amongst other issues. ${ }^{4}$ Several papers have focused on the effect of increased speed however the findings are ambiguous. For instance Foucault et al. (2013) examines the cost of being first in terms of continuous monitoring of markets while Brogaard et al. (2015) exploits a natural experiments to show that increased speed, as a result of market makers co-locating, benefits the market by enhancing liquidity. Similarly Riordan and Storkenmaier (2012) show that a decrease in latency leads to greater price efficiency and increased liquidity. In contrast Foucault et al. (2017) argue that increased speed leads to greater illiquidity and that while HFT arbitrageurs may increase price efficiency they also increase picking off risk. Menkveld and Zoican (2017) show that speeding up high frequency trading does not necessarily improve liquidity and that the effect will vary based on ratio of news to liquidity traders. There has been particular attention around the role of HFT's in flash crashes (see Kirilenko et al., 2017, for a discussion) and systemic risk e.g. Paulin et al. (2019). The best way to deal with the potentially destabilising effects of

\footnotetext{
${ }^{4}$ See Menkveld (2016) for a recent survey.
} 
high frequency trading, however, is unclear: Biais et al. (2015) argue that a tax as opposed to a ban is most beneficial to the market. Budish et al. (2015) in contrast looks at the optimal market architecture and argues that moving from continuous double auctions to discrete batch auction reduces the value of very small speed advantages and so encourages competition on price instead of speed, benefiting market participants.

Leal and Napoletano (2017) use an agent based model to consider flash crashes and the effect of several regulations. Their model is based around slow traders and HFT's who use pre-specified strategies (although slow traders may switch between chartist and fundamentalist strategies) to submit orders within trading windows of fixed length. They show how circuit breakers, cancellation fees, minimum resting times and transaction taxes affect the probability and recovery from flash crashes, notably with all but the first reducing market volatility and flash crashes but increasing the duration of these events when they occur. The model has similarities with the approach taken in this paper, however, differs in two key respects. Firstly trading strategies within this paper are endogenously determined based on the trade-off of speed and sophistication with an aim of maximising profits. Secondly trade within this paper happens within an ongoing continuous setting rather than discrete windows. As such the timing and frequency of trade is endogenous and determined by the traders own strategy and the actions of other traders, allowing traders to respond multiple times to each other in quick succession.

\section{Model}

I consider a continuous time model of the trade of a single financial asset. The asset is traded through an order book which is defined in the standard manner. It consists of a discrete series of equidistantly spaced prices at which orders may be submitted. The set of prices is $\Pi=\left\{p^{i}\right\}_{i=-\infty}^{\infty}$ whilst the distance between adjacent prices is referred to as the tick size and is equal to $\delta_{p}$. Each price has an associated queue of unfilled orders, potentially of size zero, available at that price, $l_{t}^{i}$. Each order specifies the quantity to be traded with negative values indicating sell orders and positive values representing buy orders. The set of queues together comprise the order book $L=\left\{l_{t}^{i}\right\}_{i=-\infty}^{\infty}$. The best bid is defined as the highest price at which there is an unfilled buy order $\left(B(L)=\max \left\{i \mid l^{i}>0\right\}\right)$ whilst the best ask is the lowest price at which there is an unfilled sell order $\left(A(L)=\min \left\{i \mid l_{i}<0\right\}\right)$. To address cases where no buy orders or sell orders are present,define $l^{-\infty}=1$ and $l^{\infty}=-1$. An order submitted to the book comprises a price quantity pair $(p, q)$. If an order is submitted where $q>0(q<0)$ and $p \geq A(L)(p \leq B(L))$ the order is treated as a market order and a trade occurs at the best ask (bid). If $q>0(q<0)$ and $p<A(L)(p>B(L))$ the order is treated as a limit order and added to the back of the queue $l^{p}$. As such the order book operates under price and time priority.

The fundamental value of the asset is defined as $v_{t}$ at time $t$. Innovations to the fundamental value are exponentially distributed with mean $\lambda_{v}$. Each innovation consists of the fundamental value increasing or decreasing by $\delta_{p}$ with equal probability. The initial value of the fundamental value $v_{0}$ is constrained to lie on the price grid $\Pi$ and therefore at all points in time $v_{t} \in \Pi$.

The market is populated by two groups of traders: High frequency traders who have no intrinsic value for holding the asset and slow traders who buy or sell the asset for exogenous reasons such as liquidity, hedging or speculation. 


\subsection{Slow Traders}

Slow traders are risk neutral and attempt to maximise their profits from trade. Each slow trader has a private valuation $\alpha_{i}$ relative to the fundamental value. A slow trader attempts to maximise

$$
e^{-\rho T} q_{i}\left(v_{t}+\alpha_{i}-p_{i}\right)
$$

where $p_{i}$ is the trade price and $q_{i}$ is the traded quantity. $\rho$ is a discount factor and $T$ is the length of time from the traders original entry to the market to the time of trade. The discount factor represents the cost of waiting to transact either from lost opportunities of mis-hedging costs. It does not represent interest on capital as over these timescales this would be negligible.

Slow traders arrive at the market according to an exogenous process. The times between new trader arrivals are exponentially distributed with mean $\lambda_{a}$. On arrival a trader submits an order to buy or sell a single unit of the asset. The price and direction are selected to maximise the traders profits as determined by the traders strategy (described below).

Slow traders may re-enter the market and revise unexecuted orders any number of times. The trader reenters at a random point in the future drawn from an exponential distribution with mean $\lambda_{r}$. Traders may therefore not instantly revise orders and are exposed to picking off risk. The trader may only re-enter if their original order has not executed. When a trader re-enters the market they have the option of keeping their existing order or cancelling it and submitting a new order. A trader may continue to re-enter the market and revise their order until it has executed and they leave the market forever.

\subsection{High Frequency Traders}

HFT's trade based on the order flow, buying the asset when it is under-priced and selling it when it is overpriced. $N_{h}$ HFT's are present in the market at the start and persist throughout its lifetime executing multiple trades. Initially these traders do not have an order in the market. HFT's act in one of two circumstances. HFT's may act in response to new orders from other traders as they arrive at the market. If an order arrives at the market at time $T_{0}$, an HFT responds at time $T_{0}+D_{i}$ where $D_{i}$ is the time taken for information about the arrival of an order to be transmitted to market participant $i$ through the connective technology. As such the actions of slow traders and other HFT's may stimulate an HFT trader to act which may in turn stimulate other HFT's to act. As such HFT's as a group may generate cycles of high frequency order revisions. In addition to this, like slow traders HFT's periodically re-enter the market and revise unexecuted orders. The times of these reentries are randomly determined with intervals being exponentially distributed with mean $\lambda_{r}$ and each traders random entry times being independent of all others. A new reentry time is drawn after each occasion a HFT acts. Note that $\lambda_{r}$ is much larger than $D_{i}$, therefore this random reentry plays no role in concentrated periods of HFT interaction, rather it provides the opportunity for HFT's to act during quiet periods when their is little going on in the market.

HFT traders may submit, revise orders and trade any number of times. When an order 
executes with price $p_{i}$ and quantity $q_{i}$ the HFT receives profit:

$$
e^{-\rho T} q_{i}\left(v_{t}-p_{i}\right)
$$

where $T$ is measured from the traders last trade time. Importantly unlike slow traders, fast traders have no inherent value in holding the asset. Given HFT's are always in the market they may be interpreted as maximising their rate of profits - i.e. the speed with which they make money.

\subsection{Order Submission}

Upon arriving at, or reentering, the market a trader of either type submits an order which comprises a price quantity pair $a=(p, q) . p$ is the price such that $p \in \Pi$ whilst $q$ is the quantity such that $q \in-1,0,1$. A quantity of zero represents the choice to not submit an order, in which case the value of $p$ is irrelevant. Additionally a trader with an existing unexecuted order in the market may choose to keep that order and maintain time priority.

The order submission decision is determined by the trader's strategy. Trader strategies take information about the state of the market as input and provide as output a price quantity pair as described above. The mapping between these, however, is non trivial due to the complex market architecture and interaction with other strategies. I therefore use a computational optimisation process to identify the optimal trading strategies for both groups of traders.

Traders strategies are specified through algorithms - list of instructions detailing the processing of market information to arrive at an action. These algorithms are identified through Genetic Programming - an evolutionary approach to optimisation. It works through selecting successful 'fit' strategies, in this case those that make the most money, and reproducing them in the population of candidate solutions. The reproduced strategies are subject to mutation - small perturbation's to the algorithms instructions. Over time the combination of selection, reproduction and mutation result in optimisation of the population of candidate strategies.

In this paper I use Cartesian Genetic Programming. ${ }^{5}$ Whilst there are many versions of genetic programming this approach was chosen for its speed, constrained memory usage, and interpretability. In this specification of genetic programming the programme is represented in a graph format. Each strategy has $N_{I}$ inputs which take numerical values describing the state of the world. The programme comprises $N_{N}$ nodes indexed $1, \ldots N_{N}$. Each node is a control statement or mathematical function ${ }^{6}$ which takes a number of inputs and provides a single output. For arithmetic operators this is the result of applying the operator on the two inputs. Control statement are of the form 'if then else' which compares the first two inputs and returns either the third or fourth input depending on the comparison. ${ }^{7}$ The inputs to node $k$ are either the outputs of other nodes with index $<k$ or one of the programme inputs. The outputs of the programme are taken from the values of the final two nodes.

The programme executes by evaluating the nodes from 1 to $N_{N}$ in turn. Not every node of the programme effects the result calculated. Only those nodes connected to the output (and

\footnotetext{
${ }^{5}$ See Turner and Miller, 2014 for details

${ }^{6}$ Drawn from '+', '-', 'x',' $\div$, 'abs','max','min', 'if...then'.

${ }^{7}$ Drawn from ' $>$ ', $=$ '
} 
those recursively connected to those) will have an effect. Nodes not within this set play no role. I term the length of the programme to be the number of nodes which contribute to the output. As such I assume that those nodes that do not contribute are not executed. The initial population of programmes are randomly generated. The operators and node inputs are selected at random from the available sets with equal probability.

A strategy's fitness is measured as its average payoff. For both types of traders this is the total profit obtained by traders using a strategy divided by the number of times traders using the strategy have executed orders. Reproduction occurs through genetic tournaments. Every $t_{t}$ time steps two strategies are selected at random. The strategy with lowest fitness is replaced by a copy of the strategy with higher fitness. The copied strategy is mutated. Each node and connection in the strategy undergoes a mutation with probability $p_{m}$. A mutation of a node leads to the function associated with that node being replaced by a different randomly chosen function with uniform probability. A mutation to a connection or output results in a different node or programme input being attached at random across all possibilities with equal probability. The mutated strategy has the total profit and number of orders submitted using the strategy reset to zero.

When a slow trader arrives at the market they are randomly allocated a strategy from the population of candidate slow trader strategies. They keep this strategy until their trade has executed and they leave the market. Similarly an HFT trader is randomly allocated a strategy from the population of candidate HFT strategies when they enter the market. They maintain this strategy until they have traded, at which point they are randomly allocated a new strategy. Importantly, fitness is associated with strategies rather than traders. There are at any time a fixed number of HFT's in the market, defining the level of HFT activity, however, the strategies of these HFT's will vary over time. The random selection of a strategy ensures that all candidate strategies are evaluated within the market for their potential to make profits. Separate populations are maintained for both slow and HFT traders to reflect the differing tasks they are faced with. Each population is broken down into groups - a partitioning of the the population of strategies into $N_{G}$ equally sized segments. When strategies are chosen for reproduction they are selected from the same group. This prevents the spread of one successful strategy across the whole population and supports the existence of multiple solutions if viable.

\subsection{Time}

When a trader arrives at the market at time $t$ to submit an order they observe the state of the market at time $t-D_{i}$ where $D_{i}$ is the amount of time it takes for information about the market to travel via the connective technology to the trader.

$$
D_{i}=\left\{\begin{array}{l}
t_{H}, \text { if } i=H \\
t_{S}, \text { if } i=S
\end{array}\right.
$$

where $t_{H}<t_{S}$ i.e. HFT's are closer (co-located) to the market than slow traders and receive information more quickly. Fast traders react to an order submitted to the market at time $t_{H}$ after the order has arrived, i.e. time for the information about the order to reach the trader.

When a trader chooses to submit an order, either on entry to the market or in response to 


\begin{tabular}{lcc}
\hline Parameter & Value & \\
\hline Fast traders & $N$ & {$[0-3]$} \\
Slow trader entry & $\lambda_{a}$ & 0.56 \\
Trader reentry & $\lambda_{r}$ & 5.8 \\
Fundamental innovations & $\lambda_{v}$ & 8 \\
Discount rate & $\rho$ & 0.01 \\
Tick Size & $\delta_{p}$ & 1 \\
Private values & $\alpha$ & {$[-8,-4,4,8]$} \\
Algorithm latency & $\gamma$ & 0.01 \\
Algorithm line time & $\beta$ & $2.6 \times 10^{-11}$ \\
Time to HFT's & $t_{h}$ & 0.00005 \\
Time to Slow traders & $t_{s}$ & 1 \\
Discount Rate & $r$ & 0.01 \\
Strategies & $N_{s}$ & 50 \\
Programme Length & $N_{N}$ & 100 \\
Programme Inputs & $N_{I}$ & 8 \\
Population Groups & $G$ & 4 \\
Mutation Rate & $p_{m}$ & 0.05 \\
Tournament time & $t_{t}$ & 1 \\
\hline
\end{tabular}

Table 1: Parameter used for model simulation.

receiving information about another traders order, the decision making process is not instantaneous. The time taken is dependent on the traders strategy. Longer, more complex algorithms take longer to execute and therefore the order arrives later. $E_{i}$ is the time taken for the algorithm to execute. $E_{i}=\gamma+\beta L_{i}$ where $\gamma$ is a fixed time cost to running an algorithm and $\beta$ is the time it takes to execute one line of code and $L_{i}$ is the number of lines of code in algorithm $i$. The order is then submitted and takes $D_{i}$ time to reach the market as it is transmitted over the connective technology back from the trader. An order from a trader entering the market at time $t$ will therefore enter the order book at time $t+X_{i}$ where $X_{i}=E_{i}+D_{i}$ The same approach is used for both HFT and slow traders, however, note that $D_{i}$ for slow traders is much larger in magnitude than $E_{i}$ and therefore dominates the calculation.

As a result of the delay between information being transmitted from the market, the information being processed and a resulting order arriving at the market in response $\left(2 D_{i}+X_{i}\right)$ it is possible for traders to be beaten to trading opportunities. When a trader observe a market state in which they want to submit a market order, between the observation (the point in the past at which the market was observed) and the order arriving and executing another trader could submit an order taking the same trading opportunity. This may be due to either a shorter connection time or faster algorithm. In this situation when the traders order arrives it will potentially not execute and instead enter the market as a limit order.

\subsection{Parameters}

Table 1 shows the parameterisation of the model. Four different numbers of HFT's are considered: 0-3. These represent qualitatively different scenarios: a market with no HFT's, a single HFT who can therefore be activated by slow traders only, two HFT's - the minimum for HFT's 
to activate each other and three HFT's in which there is a greater possibility for cycles of activation.

The choice of $\lambda_{a}$ and $\lambda_{r}$ are inline with previous simulation studies of high frequency order book markets such as Chiarella and Ladley (2016) and Bernales (2016). In both cases these papers use empirical data to estimate arrival and reentry times of traders. The fundamental innovation rate $\lambda_{v}$ is inline with estimates used by Goettler et al. (2009). As discussed above the discount rate, $\rho$, does not represent the risk free rate in this model rather it represents the cost of not trading and includes factors such as attention costs, missed opportunities through not executing and mis-hedging. The model was tested with other small positive values and qualitatively similar results were obtained.

Slow trader private values, $\alpha_{i}$, are drawn randomly and independently with the following support $[8,4,-4,-8] .8$ and -8 each have probability 0.2 whilst 4 and -4 have probability 0.3 . The relative sizes of these probabilities are based on the distributions estimated by Hollifield et al. (2006) for private valuations of traders in the Vancouver stock exchange. One important difference between this work and theirs is that I do not allocate any slow traders a private valuation of zero. This is because the high frequency traders implicitly have this valuation and therefore provide this component of the private value distribution.

The trading time parameters are based on empirical observation. Note that these values vary in real life between markets and technologies, I therefore take values consistent with the majority of the literature. The algorithm latency is take from Riordan and Storkenmaier (2012) and is inline with figures quoted by Menkveld and Zoican (2017). The travel time to HFT's is based on the Nasdaq estimate of round trip latency. ${ }^{8}$ The algorithmic line time is estimated from the specification of an AMD Ryzen 7 processor. Note, however, that the exact figure used here is unimportant - it is sufficient that is it much smaller than the latency, $\gamma$.

The genetic programmes have eight inputs - the current best bid and best ask prices along with the quantities available at both of these quotes. They also take the trader's private valuation, which is zero for HFT's. In addition if the trader has an order in the market they know the price, quantity and priority of that order in the execution queue. All prices in the above are measured relative to the fundamental price. This has the advantage of simplifying the trading decision by removing the level effect. As such the fundamental value is known by traders implicitly rather than as a explicit parameter.

Simulations are run for 500000 periods each of 5000 time steps. At the end of each period the fitness of all strategies is reset to zero. This helps to prevent the optimisation getting stuck in local equilibria. Each simulation is repeated 1000 times with different random seeds in order to control for randomness.

\section{Results}

The results of the model are presented in two parts. The first focuses on the strategies and profitability of HFT's in the market before considering their effect on market quality. The second group of results examines the effect of a suite of regulations on market quality and the

\footnotetext{
${ }^{8}$ See http://www.nasdaqtrader.com/Trader.aspx?id=COLO
} 


\begin{tabular}{|c|c|c|c|c|c|c|c|}
\hline Number & $\begin{array}{l}\text { Slow Tra } \\
\text { Waiting }\end{array}$ & ers -4 & $\begin{array}{l}\text { Slow Tr } \\
\text { Waiting }\end{array}$ & lers -8 & $\begin{array}{l}\text { HFT } \\
\text { Profit }\end{array}$ & $\begin{array}{l}\text { Slow } \\
\text { Lenoth }\end{array}$ & $\begin{array}{l}\text { HFT } \\
\text { Lenoth }\end{array}$ \\
\hline HFT's & Cost & Profit & Cost & Profit & & & \\
\hline 0 & $\begin{array}{l}-0.56 \\
(0.23)\end{array}$ & $\begin{array}{c}3.47 \\
(0.22)\end{array}$ & $\begin{array}{l}-1.14 \\
(0.43)\end{array}$ & $\begin{array}{c}6.70 \\
(0.38)\end{array}$ & & $\begin{array}{c}44.79 \\
(12.04)\end{array}$ & \\
\hline 1 & $\begin{array}{l}-0.43 \\
(0.20)\end{array}$ & $\begin{array}{c}3.46 \\
(0.21)\end{array}$ & $\begin{array}{l}-0.88 \\
(0.37)\end{array}$ & $\begin{array}{c}6.83 \\
(0.40)\end{array}$ & $\begin{array}{c}1.01 \\
(1.06)\end{array}$ & $\begin{array}{c}44.43 \\
(11.90)\end{array}$ & $\begin{array}{c}36.68 \\
(12.41)\end{array}$ \\
\hline 2 & $\begin{array}{l}-0.40 \\
(0.19)\end{array}$ & $\begin{array}{c}3.47 \\
(0.21)\end{array}$ & $\begin{array}{l}-0.81 \\
(0.36)\end{array}$ & $\begin{array}{c}6.89 \\
(0.40)\end{array}$ & $\begin{array}{c}0.81 \\
(0.74)\end{array}$ & $\begin{array}{c}44.55 \\
(12.43)\end{array}$ & $\begin{array}{c}30.05 \\
(11.40)\end{array}$ \\
\hline 3 & $\begin{array}{l}-0.38 \\
(0.20)\end{array}$ & $\begin{array}{c}3.44 \\
(0.25)\end{array}$ & $\begin{array}{l}-0.78 \\
(0.37)\end{array}$ & $\begin{array}{c}6.86 \\
(0.48)\end{array}$ & $\begin{array}{c}0.77 \\
(0.85)\end{array}$ & $\begin{array}{c}44.21 \\
(11.69)\end{array}$ & $\begin{array}{c}28.49 \\
(11.60)\end{array}$ \\
\hline
\end{tabular}

Table 2: Gains from trade. The table shows the average profit per trade for the three groups of traders: HFT's, Slow (valuation 4/-4) and Slow (valuation 8/-8). In addition it shows the waiting cost for each trade, defined as $\left|e^{-\rho T} \alpha\right|$ where $\mathrm{T}$ is the time between entry and trade, i.e. the reduction in potential profits from the private value as a result of time passing between the trader entering the market and executing the trade. This is always zero for HFT's due to them implicitly having a private valuation of zero. In addition for the two categories of trader, Slow and HFT, the table also shows the average length of the algorithm. All results are average over 1000 repetitions for each quantity of HFT's. Standard deviations are show in parenthesis.

behaviour of traders.

\subsection{Trading Strategies}

Previous work has ignored the effect of the trade off between speed and sophistication on the design of HFT trading strategies and their resulting success. Table 2 shows the average profits of slow and high frequency traders within the market. The presence of HFT's has very little effect on the profits of slow traders. For slow traders with a valuation of $|4|$ the average profit with or without HFT's is not statistically different. Whilst for those with a valuation of $|8|$ there is a small increase in the presence of HFT's but no statistical difference for different numbers of HFT's. In both cases the money lost due to waiting decreases, indicating that HFT's are providing a benefit through faster execution. At the same time, however, the smaller improvement in overall profits indicates that HFT's are capturing some of the gains from trade as a result of faster execution and slow traders are trading at worse prices. The result of this is that HFT's obtain positive profits per order. As the number of HFT's increases the per trade profits decrease. Competition to be first means that HFT's have to take less favourable prices, reducing gains. The impact of HFT's on slow traders is therefore positive but mixed - they provide immediacy at the cost of less favourable trading prices.

In addition to reducing HFT profits Table 2 shows that the competition between HFT's also reduces the average length of these algorithms. Increasing competition to be first to a trading opportunity results in algorithms becoming shorter and faster in order to minimise execution time. This is inline with our initial discussion. In contrast the length of slow trader algorithms is not statistically different across different specifications. The impact of the shortening in algorithm length may be seen in Figure 1. For slow traders profit per trade is increasing in the length of the algorithm up to about 40 instructions - beyond which point it flattens off. This shows that at this length there are sufficient instructions in the algorithm on average for 

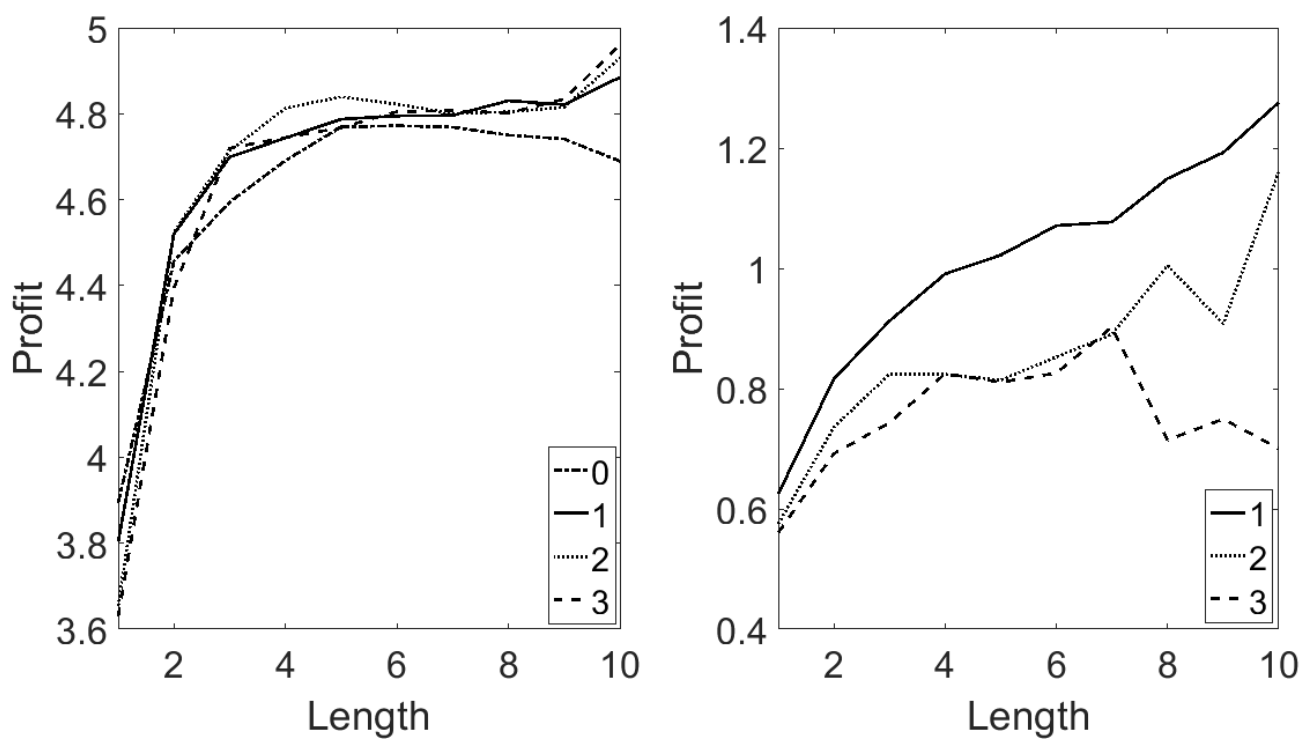

Figure 1: Average profit per order. Left - Slow traders, Right - HFT's. Results are presented for different numbers of HFT's in the market and for different algorithms lengths. For algorithm lengths, traders are assigned to 10 groups based on the number of lines of code with traders in group 1 having between 0 and 10 instructions, group 2 between 11 and 20 etc.

a trader under no pressure to shorten their algorithm to trade optimally within this setting. The average length of slow traders is greater than this value indicating that the majority of slow traders are using optimally sophisticated trading algorithms. Note, whilst some traders use shorter algorithms it does not necessarily mean these are inferior, only that they may have identified a more efficient coding but that there is no pressure for others to switch to this to achieve faster execution. For HFT's the average profit per order continues to increase. Longer algorithms are better able to identify profitable trading opportunities which shorter algorithms are not able to exploit. ${ }^{9}$

As reported in the previous section, the number of HFT's has no effect on profitability of slow traders whilst for HFT's the competition from other HFT's reduces average profits. Figure 1 shows that this is both because of the use of shorter algorithms - more traders using algorithms towards the left of each line, but also from competition, resulting in worse prices and moving the lines downwards. As such their is a dual effect, quicker less sophisticated algorithms make less profits on each trade and make fewer successful trades due to increased competition. ${ }^{10}$

Very short algorithms may suffer from an additional problem. Figure 2 shows the fraction of times an algorithm submits an order when it is has the opportunity. Very short algorithms submit orders approximately twice as often as algorithms of any other length. These algorithms may struggle to identify profitable opportunities and achieve their quickness by removing filters for unprofitable occurrences. Whilst this is necessary to be the first to trading opportunities it does give rise to greater variance in per order profits as HFT's mistakenly make trades that

\footnotetext{
${ }^{9}$ Note the problem faced by HFT's, trading without a private value, may be harder than that faced by slow traders indicating a different optimal length.

${ }^{10}$ The number of HFT algorithms in groups 9 or 10 - i.e. with long length, is relatively small increasing the variance in these points.
} 

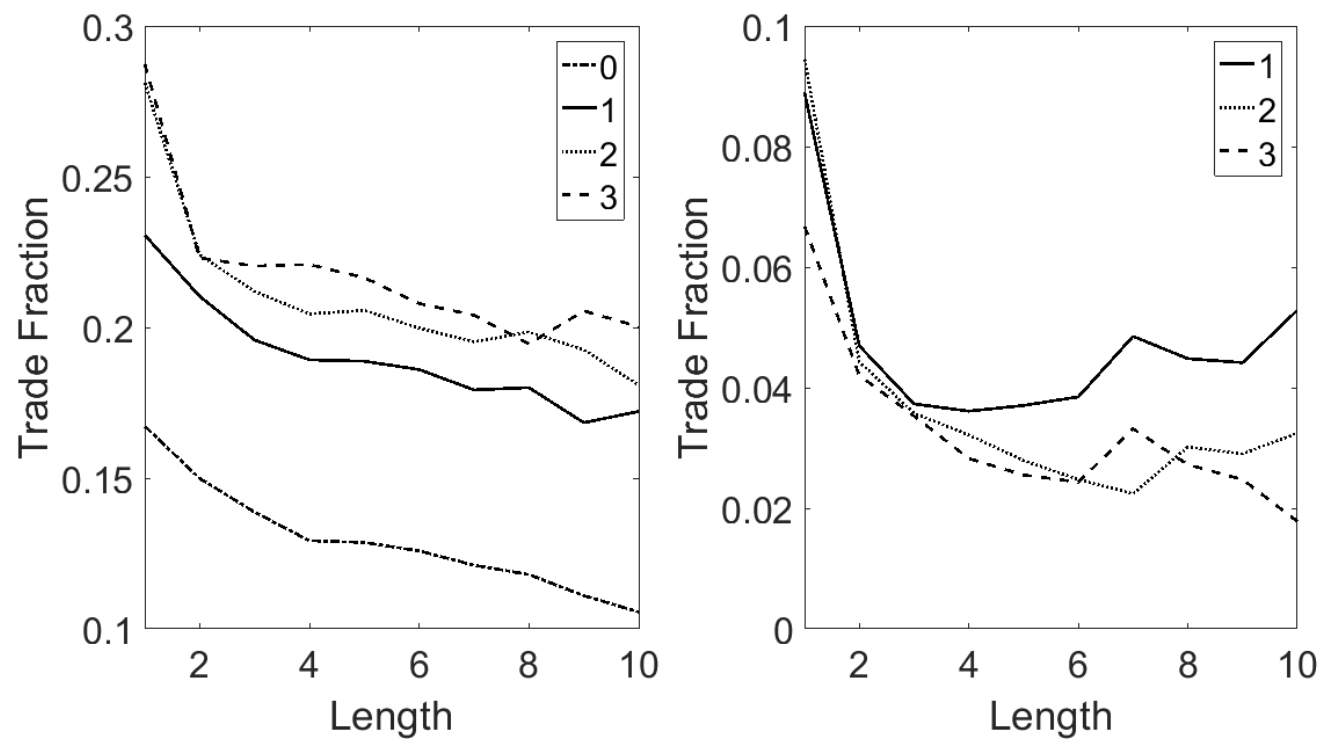

Figure 2: Submission ratio - the number of orders submitted by the trader divided by the number of chances they have to submit orders. Left - Slow traders, Right - HFT's. Results are presented for different numbers of HFT's in the market and for different algorithms lengths. For algorithm lengths, traders are assigned to 10 groups based on the number of lines of code with traders in group 1 having between 0 and 10 instructions, group 2 between 11 and 20 etc.

result in low profits in some cases. The positive effect of HFT's on slow traders may also be seen in Figure 2 as greater numbers of HFT's increase the fraction of slow trader orders that execute, therefore reducing weighting costs.
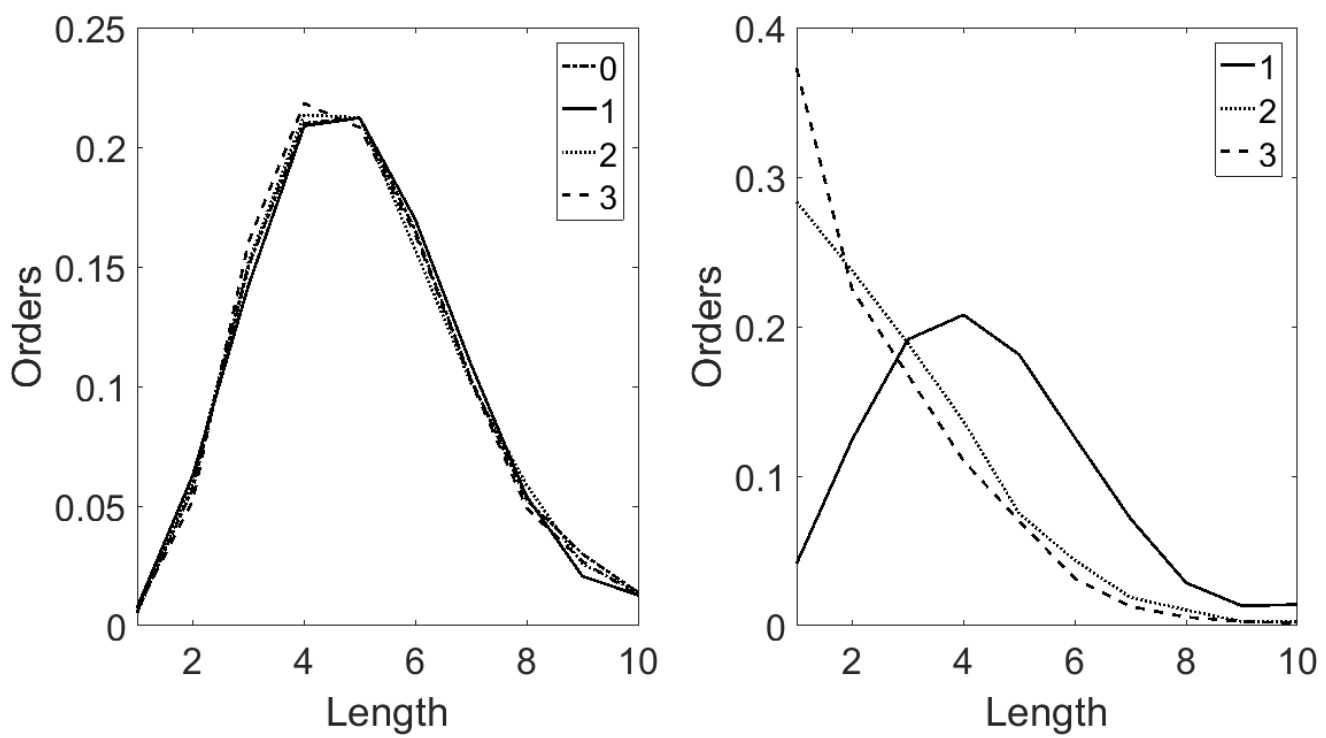

Figure 3: Fraction of orders submitted by traders. Left - Slow traders, Right - HFT's. Results are presented for different numbers of HFT's in the market and for different algorithms lengths. Fraction of orders is the number of orders submitted by traders of that type and length divided by the total number submitted by that type across all strategy lengths. For algorithm lengths, traders are assigned to 10 groups based on the number of lines of code with traders in group 1 having between 0 and 10 instructions, group 2 between 11 and 20 etc. 


\begin{tabular}{cccccc}
\hline $\begin{array}{c}\text { Number } \\
\text { HFT's }\end{array}$ & Spread & $\begin{array}{c}\text { Best Ask } \\
\text { Quantity }\end{array}$ & $\begin{array}{c}\text { Total Ask } \\
\text { Quantity }\end{array}$ & Volatility & $\begin{array}{c}\text { Pricing } \\
\text { Error }\end{array}$ \\
\hline 0 & 2.62 & 10.0 & 61.6 & 0.85 & 2.25 \\
& $(0.51)$ & $(5.4)$ & $(55.8)$ & $(0.36)$ & $(0.55)$ \\
1 & 2.34 & 7.7 & 42.0 & 0.90 & 1.79 \\
& $(0.44)$ & $(4.5)$ & $(47.8)$ & $(0.28)$ & $(0.57)$ \\
2 & 2.15 & 7.0 & 36.1 & 0.91 & 1.60 \\
& $(0.48)$ & $(4.0)$ & $(40.0)$ & $(0.28)$ & $(0.58)$ \\
3 & 2.02 & 6.9 & 38.3 & 0.89 & 1.52 \\
& $(0.52)$ & $(4.5)$ & $(45.8)$ & $(0.24)$ & $(0.66)$ \\
\hline
\end{tabular}

Table 3: Market quality. All results are average over 1000 repetitions for each quantity of high frequency traders. All distance measures are in ticks whilst quantity measures are in units. The quantities at the best bid and in the buy book are similar to those at the best ask and in the ask book. Volatility is measured in ticks over 10 minute in model periods. Pricing error is the average absolute distance between the mid point of the spread and the fundamental value. Standard deviations are in parenthesis.

Figure 3 shows the number of orders submitted by each type of trader conditional on algorithm length. For slow traders the curves overlap and show that most traders have a very similar algorithm length. There is no competition over algorithm speed and therefore no incentive to shorten algorithms below the approximately 40 instructions needed to trade effectively. For HFT's the number of orders submitted by different algorithms differs markedly for different market configurations. When there is only a single HFT the distribution is similar in shape to that of slow traders - i.e. indicating no incentive to shorten the length of code. The introduction of a second HFT, however, changes this markedly. Instead of peaking around 40 instructions algorithms with 0-10 instructions are seen to submit the most orders with a monotonic decrease in orders submitted as length increases. A further HFT makes this pattern more pronounced with an even greater share of orders being submitted by very short algorithms. This clearly demonstrates competition on algorithmic speed. The introduction of a second competitor algorithm pushes traders to reduce the length of their code with further traders increasing this pressure. It is important to highlight that the differences in execution time are very small as the line execution time is $2.6 \times 10^{-11}$. Whilst the effect on the absolute time for execution is small, the relative effect - being shortest and therefore first to the trading opportunity, is crucial to success. The strategy of these short HFT's is clear, taken together with those results in Figure 1, these traders make large numbers of trades with relatively small profits. In contrast those HFT's who use longer algorithms submit fewer orders but make greater profits per trade. As such their is a continuum of viable HFT strategies ranging from quick and unsophisticated to slower and intelligent all of which make profits. The distribution of traders types within the market effects the relative mix of strategies in equilibrium.

\subsection{Market Quality}

The effect of HFT's on the functioning of markets has received much interest, in particular the role they play on liquidity, price efficiency and stability are all areas of discussion. Table 3 presents results showing the effect of different levels of high frequency trading on market quality. High frequency traders are shown to have an ambiguous effect on liquidity - they narrow the 
spread but also reduce the quantities available both at the best quotes and throughout the book as a whole. This agrees with the empirical observations of Korajczyk and Murphy (2018) who find that a reduction in HFT's widens spreads and reduces price impact. HFT's are able to respond to information more quickly than slow traders, as a result they are able to narrow the quotes without being subject to the same degree of picking off risk and at the same time are able to quickly match any orders from other traders that become stale. The presence of multiple HFT's also creates competition forcing HFT's and other traders to shave their prices and narrow the spread in order to trade. This ability to respond quickly to information and maintain a narrow spread results in lower average pricing error - the market better tracks the fundamental. Importantly, whilst the average error goes down the variance in the pricing error goes up. This is because groups of HFT's can respond to each others order's repeatedly and so generate quote churn which is not associated with information or trading needs.

This effect is also evidenced in the volatility as measured by changes in the mid price. The presence of HFT's leads to an increase in volatility from the adjustment of quotes and picking off of orders by HFT's responding quickly to the changing market. Whilst HFT's increase volatility, the number of HFT's has no statistically significant effect. The presence of only one HFT is sufficient to observe this effect. One HFT is able to respond to every action within the market whilst multiple HFT's respond to each other. Volatility, however, is only measured at set intervals these very fast HFT interactions have little effect on the market dynamics as measured by volatility.

\section{Regulation}

There has been much debate about the regulation of financial markets in the presence of HFT's. In particular HFT's have been accused of damaging market quality and inhibiting trading opportunities for other traders. As a result, a suite of regulations have been proposed to limit the dangers of HFT's and their participation in the market or to drive them from the market all together. In this section I consider six possibilities.

\subsection{Specification}

Each regulation comprises a modification of the above model above. I consider each regulation separately versus the case of a market comprising three HFT's and no regulations.

\subsubsection{Make/Take Fee}

Make take fees have been common within markets for a number of years and serve as an important benchmark for understanding the role of market design and regulation on HFT's. They have been shown to effect the behaviour of HFT's and therefore the market liquidity (Foucault et al., 2013). Make take fees work by imposing a costs $\zeta$ on the submitter of a market order (whether that is an order to buy or sell) and at the same time by paying a limit order supplier who's order successfully executes a fee of $\zeta$ (again regardless of whether they are a buyer or seller). The market order fee is often larger than the limit order supplier fee in order to provide a small profit to the exchange, however, in this model we abstract from this assumption. 


\subsubsection{Tick Size}

High frequency traders often make money through offering small improvements on the best quotes. In order to limit their ability to do this it has been proposed to increase the tick size. This effectively increases the cost to improving on the best quotes. Within the base model we take a tick size $\delta_{p}=1$. Under this regulation the tick size in increased. This means that all orders may only be placed on a price grid in which prices are separated by larger increments. Note in order that the fundamental value also remains on the ticks the size of fundamental changes is increased to match the new tick size whilst rate of innovations is reduced such that the fundamental volatility remains the same as the base case.

\subsubsection{Speed Bump}

One proposed method to reduce the speed advantage of HFT's is to delay the arrival time of their orders. This approach is frequently referred to as a speed bump. The effect of this is to limit the benefits of being fast, as despite reacting to information quickly their is delay in acting. Without the regulation an order submitted at time $t$ reaches the order book at time $t+D_{i}$ where $D_{i}$ is a fixed time dependent on the class of traders. With this regulation the order arrival time is: $t+D_{i}+\psi$ where $\psi$ is the time taken for the arriving order to cross the speed bump. Note the speed bump does not effect the speed of dissemination of information going in the opposite direction.

\subsubsection{Minimum Resting Time}

An alternative way to slow down and limit the advantage of HFT's is to limit how quickly they can change the orders submitted to the book. Rather than delaying orders arriving at the book it has been proposed to require orders submitted by traders to spend a minimum amount of time resting in the book before they can be changed or cancelled. I refer to this minimum resting time as $\omega$. Within the model an order cannot be changed until $\omega$ seconds have passed since the order reached the book. In practical terms a high frequency trader $i$, submitting an order at time $t$, will next become active and look at their order at time $t+\omega-D_{i}-E_{i}$. This is the time such that the trader will be able to change their order at the first available time point after the order becomes available for modification.

\subsubsection{Order Ratio}

High frequency traders often rapidly change their orders to respond to other HFT's and changes in the market. In order to limit this advantage of HFT's and make the market more transparent several exchanges limit the ratio of orders an HFT can submit relative to the number of trades (Menkveld, 2013, see for instance Chi-X as discussed by). Within this model this calculation is performed for each trading algorithm. At its creation, either at the start of the simulation or through a tournament, algorithm $i$ 's count of trades, $\xi_{i}^{M}$, and orders, $\xi_{i}^{L}$, are set to 0 . Every order a trader submits, whether a market or limit order, is added to the respective counts. A trader may only submit a limit order if the ratio $\frac{\xi_{i}^{M}}{\xi_{i}^{L}}>\Xi$, otherwise they must submit a market order, guaranteeing a trade, or take no action. 


\begin{tabular}{lcc}
\hline Parameter & Value & \\
\hline Make/Take Fee & $\zeta$ & 0.5 \\
Tick Size & $\delta$ & 2 \\
Speed Bump & $\psi$ & 1.0 \\
Rest Time & $\omega$ & 1.0 \\
Order Ratio & $\Xi$ & 0.2 \\
Tax & $\tau$ & 0.5 \\
\hline
\end{tabular}

Table 4: Regulatory parameters and their definitions.

\subsubsection{Transaction Tax}

A transaction tax is a tax on each participant in a financial trade. It has a long history in finance since it was proposed by Tobin in 1972, see Lensberg et al. (2015) for an example of its effect in an order book market. In this paper I consider a tax at a fixed rate $\tau$ which is subtracted from the profits of both the buyer and seller when a trade occurs. The payoff of a slow trader, Equation 3.1 is thus modified as:

$$
e^{-\rho T} q_{i}\left(v_{t}+a l p h a_{i}-p_{i}\right)-\tau
$$

Whilst fast trader payoffs are modified analogously.

\section{$5.2 \quad$ Results}

Each of the regulations described above is considered separately. In all cases the functioning of the regulation is governed by a parameter value shown in Table 4 . The parameter values chosen can be varied in order to test different levels or strengths of the regulation. Results are presented for empirically plausible levels, however, they have been tested for a range of other parameter values to ascertain that the qualitative effects are robust. In the analysis I focus on the effect and comparison of each regulation with the base case and qualitative comparison of the effects between regulations. I avoid, where possible, making quantitative comparisons between regulations as these can be influenced by the choice of regulatory parameters.

Table 5 shows the effect of the six regulations on market quality. Make take fees and increased tick sizes have an ambiguous effect on liquidity within the market - widening the spread and increasing the available quantity. In each case the wider spread increases the costs to using market orders and trading therefore increasing the use of limit orders, decreasing trade and increasing the available quantity in the books. Tick sizes do this mechanically be forcing greater gaps in the price grid. Make take fees, have a similar effect by making it more expensive to initiate a trade and increasing the incentive to be the limit order supplier relative to the liquidity taker. The increased preference for limit orders over market orders leads to both slow and HFT traders spending longer in the market prior to executing. The larger quantities at the quotes lead to fewer changes in price and therefore lower volatility under make-take fees, however, a larger tick size mechanically increases this measure. Similarly the reluctance to use market orders means that the market is slower to track changes in the fundamental price.

Like increases in tick size and make take fees, speed bumps slightly increase available liquidity 


\begin{tabular}{lccccccc}
\hline & & Best Ask & Total Ask & & Pricing & Slow & HFT \\
Regulation & Spread & Quantity & Quantity & Volatility & Error & Time & Time \\
\hline Base & 2.02 & 6.91 & 38.27 & 0.89 & 1.52 & 3.26 & 0.20 \\
& $(0.52)$ & $(4.45)$ & $(45.78)$ & $(0.24)$ & $(0.66)$ & $(0.23)$ & $(0.07)$ \\
Make/Take Fee & 2.28 & 8.73 & 54.54 & 0.85 & 1.91 & 3.39 & 0.23 \\
& $(0.51)$ & $(4.99)$ & $(54.69)$ & $(0.30)$ & $(0.62)$ & $(0.19)$ & $(0.10)$ \\
Tick Size & 3.82 & 16.34 & 124.59 & 1.25 & 2.84 & 3.47 & 0.21 \\
& $(1.35)$ & $(14.87)$ & $(153.42)$ & $(0.70)$ & $(1.42)$ & $(0.28)$ & $(0.13)$ \\
Speed Bump & 2.06 & 7.41 & 40.43 & 0.88 & 1.68 & 4.65 & 1.43 \\
& $(0.43)$ & $(4.46)$ & $(49.68)$ & $(0.27)$ & $(0.61)$ & $(0.09)$ & $(0.22)$ \\
Resting Time & 2.04 & 7.15 & 37.61 & 0.86 & 1.56 & 3.30 & 0.32 \\
& $(0.45)$ & $(4.26)$ & $(47.17)$ & $(0.23)$ & $(0.59)$ & $(0.18)$ & $(0.11)$ \\
Order Ratio & 2.48 & 2.27 & 9.87 & 1.32 & 1.18 & 2.26 & 0.09 \\
& $(1.08)$ & $(1.50)$ & $(13.02)$ & $(0.48)$ & $(0.43)$ & $(0.24)$ & $(0.04)$ \\
Tax & 1.84 & 5.35 & 29.39 & 1.01 & 1.15 & 3.12 & 0.13 \\
& $(0.77)$ & $(3.99)$ & $(41.75)$ & $(0.37)$ & $(0.70)$ & $(0.27)$ & $(0.06)$ \\
\hline
\end{tabular}

Table 5: Market quality under regulation. All results are average over 1000 repetitions for each regulation. All distance measures are in terms of ticks whilst quantity measures are in units. The quantities at the best bid and in the buy book are similar to those at the best ask or in the ask book. Volatility is measured in ticks over 10 minute in model periods. Pricing error is the average absolute distance between the mid point of the spread and the fundamental value. Slow time and HFT time are the average amount of time for a slow trader to trade from the point it enters and the average time between HFT trades. Standard deviations are in parenthesis.

at the best quote and in the book but do not have a significant effect on the spread or volatility. The additional risk of orders being stale when they arrive due to changes in the fundamental value between submission and arrival encourages slightly less aggressive order submissions, however, this risk is small so the effect is minor. The only economically significant change between this and the base case is the increase in time to trade which is a direct effect of the delay in orders reaching the market. Minimum resting times do not have any significant effect on either liquidity measure, volatility or pricing accuracy. Whilst they do increase picking off risk to some extent this occurs across all traders and apart from a small increase in the time HFT's take to trade the result in no net effect.

Transaction taxes have the opposite effect to the above regulations - reducing the spread and the quantity available. In this case the regulation increases the cost of all traders. This pushes traders to be more aggressive (using more market orders and limit orders within the spread) in order to minimise their time in the market and waiting costs. As a result there is less quantity available in the book but traders tend to narrow the spread to increase the probability of a quick trade. This aggressiveness has the benefit of increasing the pricing accuracy of the market but at the expense of increasing volatility. An order ratio, however, unambiguously damages market quality. It increases volatility and pricing error and reduces liquidity - both widening the spread and reducing quantity available in the book. In this case the regulation discourages traders, particularly HFT's from submitting limit orders - regardless of aggressiveness - and forces them to submit market orders to maintain their order ratio. As a result less quantity enters the book and it is more quickly removed by traders using market orders. This effect is inline with the empirical results of Friederich and Payne (2015) who show that the introduction of a similar 


\begin{tabular}{|c|c|c|c|c|c|}
\hline \multirow[b]{2}{*}{ Regulation } & \multicolumn{2}{|c|}{$\begin{array}{l}\text { Slow Traders - } 4 \\
\text { Waiting }\end{array}$} & \multicolumn{2}{|c|}{$\begin{array}{l}\text { Slow Traders - } 8 \\
\text { Waiting }\end{array}$} & \multirow{2}{*}{$\begin{array}{c}\text { HFT Traders } \\
\text { Profit }\end{array}$} \\
\hline & Cost & Profit & Cost & Profit & \\
\hline \multirow[t]{2}{*}{ Base } & -0.38 & 3.44 & -0.78 & 6.86 & 0.77 \\
\hline & $(0.20)$ & $(0.25)$ & $(0.37)$ & $(0.48)$ & $(0.85)$ \\
\hline \multirow[t]{2}{*}{ Make/Take Fee } & -0.47 & 3.44 & -0.96 & 6.75 & 0.90 \\
\hline & $(0.21)$ & $(0.23)$ & $(0.40)$ & $(0.45)$ & $(1.16)$ \\
\hline \multirow[t]{2}{*}{ Tick Size } & -0.45 & 3.44 & -1.05 & 6.60 & 1.48 \\
\hline & $(0.30)$ & $(0.48)$ & $(0.62)$ & $(0.67)$ & $(2.31)$ \\
\hline \multirow[t]{2}{*}{ Speed Bump } & -0.47 & 3.39 & -0.95 & 6.72 & 0.78 \\
\hline & $(0.17)$ & $(0.22)$ & $(0.33)$ & $(0.44)$ & $(0.84)$ \\
\hline \multirow[t]{2}{*}{ Minimum Rest } & -0.40 & 3.47 & -0.81 & 6.90 & 0.87 \\
\hline & $(0.17)$ & $(0.19)$ & $(0.33)$ & $(0.38)$ & $(0.72)$ \\
\hline \multirow[t]{2}{*}{ Order Ratio } & -0.17 & 3.58 & -0.33 & 7.28 & 0.05 \\
\hline & $(0.10)$ & $(0.25)$ & $(0.15)$ & $(0.43)$ & $(0.07)$ \\
\hline \multirow[t]{2}{*}{ Tax } & -0.31 & 2.95 & -0.61 & 6.49 & 0.42 \\
\hline & $(0.18)$ & $(0.23)$ & $(0.31)$ & $(0.44)$ & (1.07) \\
\hline
\end{tabular}

Table 6: Gains from trade under regulation. The table shows the average profit per trade for the three groups of traders: HFT's, Slow (valuation $4 /-4$ ) and Slow (valuation $8 /-8$ ). In addition it shows the waiting cost for each trade, defined as $e^{-\rho T} \alpha$ where $\mathrm{T}$ is time between entry and trade, i.e. the reduction in potential profits from the private value as a result of time passing between the trader entering the market and executing the trade. All results are average over 1000 repetitions for each quantity of HFT's. Standard deviations are shown in parenthesis.

rule in the Italian market harmed liquidity. This differs, however from the findings of Jorgensen et al. (2018) who show no effect on liquidity for a similar regulation in the Oslo stock exchange. The principal difference in these two cases is that the latter had features designed to encourage liquidity provision. The rule we test is much closer to that of the first for which the findings are in broad agreement.

Table 6 presents the profits of both slow and HFT traders under a range of regulations. As has previously been suggested by the literature make/take fees benefit HFT's. In particular they are able to benefit from the payments for providing liquidity whilst, due to their speed advantage, they are able to avoid much of the risk. This benefit comes at the expenses of slow traders, particularly those with the highest value units who obtain lower profits. The immediacy that these traders typically desire costs them money in the form of market orders fees which are transferred to HFT's. Increasing the tick size similarly benefits HFT's at the expense of slow traders with high value units. In this case the larger price grid means that the cost of being picked off increases whilst the benefits to picking another order off similarly increase. This benefits HFT's who are better able to avoid the first and exploit the second case.

Speed bumps do not have a positive effect - they reduce the profits of slow traders whilst leaving HFT's unaffected. Minimum resting times are beneficial to all traders to some extent. Small but statistically significant increases in per order profits are observed for both groups of slow traders whilst HFT's observe a greater increase in profits. For HFT's, however, this is accompanied by an increase in the variance of per order profits. Insisting on minimum resting times for orders means more opportunities to pick off other traders quote's and make greater 
profits. At the same time in some cases HFT's will have stale orders that will be picked off by slower traders, or other HFT's who arrive at the right point in time. Whilst minimum resting times and speed bumps work in a similar manner, the former is more beneficial to both groups of traders. This difference is due to where the delay occurs. In the case of speed bumps it is prior to the order arriving at the market - effectively increasing waiting costs, whilst for minimum resting times the same delay in arrival is not present.

If the focus of regulation were to drive high frequency traders from markets, despite their positive effects, transaction taxes and order ratio's both reduce profits for this group. Transaction taxes do this by increasing the cost to trading. This negatively effects HFT's by reducing or completely eliminating the small profits they make on each order. This same effect, however, also applies to slow traders and reduces the profits of non HFT traders by a greater amount. Order ratio's in contrast have the dual advantage of slightly increasing non-HFT trader profits whilst making HFT trading almost completely unprofitable. The order ratio makes the normal strategy of HFT's based on rapidly adjusting limit orders impossible to maintain. The requirement to make a fraction of trades relative to orders effectively eliminates their ability to use speed to provide the best quotes at all times. This regulatory change completely negates the advantage of HFT traders. The order ratio has little direct effect on non-HFT's as they typically use market orders more frequently. This would therefore appear to be a very effective tool in regulating HFT's profits but with the non-trivial effects on market quality noted above.

\section{Conclusion}

The theoretical analysis of high frequency trading has generally focused on optimal behaviour, and so has ignored the trade-off between sophisticated decision making and time. I present a model of the interaction of high frequency traders in which trading speed is dictated by strategy sophistication and the choice of strategy sophistication is endogenous. Using this model I show that there are multiple equilibria in strategy design. Despite competition to be first by being quickest, traders adopt a range of sophistication's with short strategies making large numbers of low profit trades and more sophisticate strategies identifying a smaller number of high profit trades. A suite of regulations proposed to limit the negative effects of HFT's are shown to generally be ineffective in either improving market quality or reducing the profits of HFT's. The only exception is that of a ratio for the number of trades to orders, this may benefit slow traders at the expense of HFT' but with some negative consequences on the market as a whole. 


\section{References}

Bernales, A., 2016. Algorithmic and high frequency trading in dynamic limit order markets. Tech. rep., SSRN.

Biais, B., Foucault, T., Moinas, S., 2015. Equilibrium fast trading. Journal of Financial Economics 116 (2), 292-313.

Breaban, A., Noussair, C. N., 2015. Trader characteristics and fundamental value trajectories in an asset market experiment. Journal of Behavioral and Experimental Finance 8, 1 - 17.

Brogaard, J., Hagstrmer, B., Nordn, L., Riordan, R., 2015. Trading fast and slow: Colocation and liquidity. The Review of Financial Studies 28 (12), 3407-3443.

Brogaard, J., Hendershott, T., Riordan, R., 2014. High-frequency trading and price discovery. The Review of Financial Studies 27 (8), 2267-2306.

Budish, E., Cramton, P., Shim, J., 2015. The High-Frequency Trading Arms Race: Frequent Batch Auctions as a Market Design Response. The Quarterly Journal of Economics 130 (4), $1547-1621$.

Burks, S. V., Carpenter, J. P., Goette, L., Rustichini, A., 2009. Cognitive skills affect economic preferences, strategic behavior, and job attachment. Proceedings of the National Academy of Sciences 106 (19), 7745-7750.

Chen, S.-H., Tai, C.-C., Yang, L.-X., 2017. Cognitive capacity and earnings performance: Evidence from double auction market experiments. Tech. rep., mimeo.

Chen, Y., Wang, X., 2015. A hybrid stock trading system using genetic network programming and mean conditional value-at-risk. European Journal of Operational Research 240 (3), 861 871.

Chiarella, C., Ladley, D., 2016. Chasing trends at the micro-level: The effect of technical trading on order book dynamics. Journal of Banking \& Finance 72 (S), 119-131.

Corgnet, B., Hernán-González, R., Kujal, P., Porter, D., 2015. The effect of earned versus house money on price bubble formation in experimental asset markets. Review of Finance 19 (4), $1455-1488$.

Coval, J. D., Hirshleifer, D., Shumway, T. G., Dec. 2004. Can Individual Investors Beat the Market? Finance 0412005, EconWPA.

Delaney, L., 2018. Investment in high-frequency trading technology: A real options approach. European Journal of Operational Research 270 (1), 375 - 385.

Foucault, T., Kadan, O., Kandel, E., 2013. Liquidity cycles and make/take fees in electronic markets. The Journal of Finance 68 (1), 299-341.

Foucault, T., Kozhan, R., Tham, W. W., 2017. Toxic Arbitrage. Review of Financial Studies 30 (4), 1053-1094. 
Friederich, S., Payne, R., 2015. Order-to-trade ratios and market liquidity. Journal of Banking \& Finance 50, $214-223$.

Goettler, R. L., Parlour, C. A., Rajan, U., 2009. Informed traders and limit order markets. Journal of Financial Economics 93 (1), $67-87$.

Grinblatt, M., Keloharju, M., Linnainmaa, J. T., 2012. IQ, trading behavior, and performance. Journal of Financial Economics 104 (2), 339-362.

Hagströmer, B., Nordén, L., 2013. The diversity of high-frequency traders. Journal of Financial Markets 16 (4), $741-770$.

Hendershott, T., Jones, C. M., Menkveld, A., 2011. Does algorithmic trading improve liquidity? Journal of Finance 66 (1), 1-33.

Hollifield, B., Miller, R. A., Sandås, P., Slive, J., 2006. Estimating the gains from trade in limit-order markets. The Journal of Finance 61 (6), 2753-2804.

Jorgensen, K., Skjeltorp, J., degaard, B. A., 2018. Throttling hyperactive robots order-to-trade ratios at the oslo stock exchange. Journal of Financial Markets 37, 1- 16.

Kirilenko, A., Kyle, A. S., Samadi, M., Tuzun, T., 2017. The flash crash: High-frequency trading in an electronic market. The Journal of Finance 72 (3), 967-998.

Korajczyk, R. A., Murphy, D., 07 2018. High-Frequency Market Making to Large Institutional Trades. The Review of Financial Studies 32 (3), 1034-1067.

Ladley, D., Lensberg, T., Palczewski, J., Schenk-Hopp, K. R., 2015. Fragmentation and stability of markets. Journal of Economic Behavior \& Organization 119 (C), 466-481.

Leal, S. J., Napoletano, M., 2017. Market stability vs. market resilience: Regulatory policies experiments in an agent-based model with low- and high-frequency trading. Journal of Economic Behavior and Organization.

Lensberg, T., Schenk-Hopp, K. R., Ladley, D., 2015. Costs and benefits of financial regulation: Short-selling bans and transaction taxes. Journal of Banking and Finance 51, 103 - 118.

Manahov, V., Soufian, M., Hudson, R., 01 2014. The Implications Of Trader Cognitive Abilities On Stock Market Properties. Intelligent Systems in Accounting, Finance and Management $21(1), 1-18$.

Menkveld, A., Zoican, M. A., 2017. Need for Speed? Exchange Latency and Liquidity. Post-Print hal-01501352, HAL.

Menkveld, A. J., 2013. High frequency trading and the new market makers. Journal of Financial Markets 16 (4), $712-740$.

Menkveld, A. J., October 2016. The Economics of High-Frequency Trading: Taking Stock. Annual Review of Financial Economics 8 (1), 1-24. 
Moore, H. L., 1911. Laws of wages; an essay in statistical economics. New York: The Macmillan Company.

Neely, C., Weller, P., Dittmar, R., 1997. Is technical analysis in the foreign exchange market profitable? a genetic programming approach. Journal of Financial and Quantitative Analysis $32(4), 405426$.

Noussair, C. N., Tucker, S., Xu, Y., 2016. Futures markets, cognitive ability, and mispricing in experimental asset markets. Journal of Economic Behavior \& Organization 130 (C), 166-179.

Paulin, J., Calinescu, A., Wooldridge, M., 2019. Understanding flash crash contagion and systemic risk: A micromacro agent-based approach. Journal of Economic Dynamics and Control $100,200-229$.

Riordan, R., Storkenmaier, A., 2012. Latency, liquidity and price discovery. Journal of Financial Markets 15 (4), $416-437$.

Turner, A. J., Miller, J. F., 2014. Introducing a cross platform open source cartesian genetic programming library. The Journal of Genetic Programming and Evolvable Machines 16, 8391.

Yeh, C.-H., 2008. The effects of intelligence on price discovery and market efficiency. Journal of Economic Behavior and Organization 68 (3), $613-625$.

Yeh, C.-H., Yang, C.-Y., 2010. Examining the effectiveness of price limits in an artificial stock market. Journal of Economic Dynamics and Control 34 (10), 2089 - 2108. 